\title{
P-V Criticality of a Modified BTZ Black Hole in 2 + 1 Dimensional Intrinsic Time Quantum Gravity
}

\author{
A. S. Kubeka \\ Department of Mathematical Sciences, University of South Africa, Science Campus, Florida, South Africa \\ Email: kubekas@unisa.ac.za
}

How to cite this paper: Kubeka, A.S. (2019) P-V Criticality of a Modified BTZ Black Hole in $2+1$ Dimensional Intrinsic Time Quantum Gravity. Journal of Modern Physics, 10, 294-301.

https://doi.org/10.4236/jmp.2019.103020

Received: December 14, 2018

Accepted: March 8, 2019

Published: March 11, 2019

Copyright (อ 2019 by author(s) and Scientific Research Publishing Inc. This work is licensed under the Creative Commons Attribution International License (CC BY 4.0).

http://creativecommons.org/licenses/by/4.0/

\begin{abstract}
Intrinsic time quantum geometrodynamics is a formulation of quantum gravity naturally adapted to $3+1$ dimensions. In this paper we construct its analogous $2+1$ formulation, taking note of the mathematical structures which are preserved. We apply the resulting construction to convert the BTZ black hole metric to ITQG framework. We then modify the BTZ black hole in order to investigate the existence of the P-V criticality in ITQG theory.
\end{abstract}

\section{Keywords}

Intrinsic Time Quantum Geometrodynamics, Modified BTZ Black Hole, $2+1$ Quantum Gravity, Black Hole P-V Criticality, Van der Waals Fluid, Liquid-Gas Phase Transition

\section{Introduction}

P-V criticality of a modified BTZ black hole was recently studied by Kubeka and Sadeghi in [1]. They introduced some anstaz that gives a modified BTZ black hole and then showed that this modified BTZ black hole is satisfied by the equation of state of Liquid-gas phase transition. Also Sadeghi [2] studied the P-V criticality of Logarithmic corrected Dyonic charged AdS black hole by considering a charged AdS black hole which is in fact a holographic dual of a van der Waals fluid. They showed that the holographic picture is still valid. The logarithmic corrections arise due to the fluctuations in thermal properties which are important when the black hole is small and these fluctuations are interpreted as quantum effects.

The theory of quantum Gravity that encompasses the notion of intrinsic time 
(Intrinsic Time Quantum Geometrodynamics, ITQG) was recently formulated by Ita et al. [3]. The foundational work for the theory was laid down in [4]-[10]. In [11], Ita et al. solved the long outstanding Wheeler-DeWitte equation in Quantum Geometrodynamics and derived cosmological exact solutions that are characterized by the Schrodinger wave functionals that in turn have two physical degrees of freedom in accordance with the Yamade problem in quantum gravity. The theory seems to resolve all the outstanding problems in quantum gravity that have been highlighted by other prominent theories of quantum gravity like Loop Quantum Gravity (LQG) in one single stroke and in clean manner Ita et al. [12]. The success and validity of this theory will be tested by future research.

In this paper we study the transform of the modified BTZ black hole into the ITQG formalism and then establish the existence of the P-V criticality as done in the literature. The paper is structured as follow: In Sec. 2, we reformulate the ITQG formalism in 2-dimension, and in Sec. 3, we reformulate the BTZ black hole within the context of ITQG. In Sec. 4, we study and establish the existence of the P-V criticality of a modified BTZ black in ITQG theory, and then lastly in Sec. 5 conclude the paper.

\section{Quantum Gravity in 2+ 1 Dimension}

In [3] a formulation of intrinsic time quantum geometrodynamics (ITQG) is presented, which is applicable to $3+1$ dimensional spacetime. We would like to study an analogous $2+1$ formulation corresponding to two spatial and one temporal dimension. There are a few similarities and differences which will be noted in this paper. Let us start off by taking, as the basic variables, a unimodular spatial two-metric $\bar{q}_{i j}$ with $\operatorname{det} \bar{q}_{i j}=1$ and a mixed-index, traceless, momentric variable $\bar{\pi}_{j}^{i}$ with $\delta_{i}^{j} \bar{\pi}_{j}^{i}=0$. For index conventions in this paper, lower case Latin symbols $i, j, \cdots$ will denote spatial indices in two-space, taking values 1 and 2. Upon these variables let us confer the following commutation relations

$$
\begin{aligned}
& {\left[\bar{q}_{i j}(x), \bar{q}_{k l}(y)\right]=0 ;\left[\bar{q}_{i j}(x), \bar{\pi}_{l}^{k}(y)\right]=i \hbar \bar{E}_{l(i j)}^{k} \delta(x-y) ;} \\
& {\left[\bar{\pi}_{j}^{i}(x), \bar{\pi}_{l}^{k}(y)\right]=\frac{i \hbar}{2}\left(\delta_{l}^{i} \bar{\pi}_{j}^{k}-\delta_{j}^{k} \bar{\pi}_{l}^{i}\right) \delta(x-y),}
\end{aligned}
$$

where $\delta(x-y)$ denotes the two dimensional delta function, and

$\bar{E}_{l(i j)}^{k}=\frac{1}{2}\left(\delta_{i}^{k} \bar{q}_{l j}+\delta_{j}^{k} \bar{q}_{l i}-\delta_{l}^{k} \bar{q}_{i j}\right)$ is a traceless projector, with

$\delta_{k}^{l} \bar{E}_{l(i j)}^{k}=\bar{q}^{i j} \bar{E}_{l(i j)}^{k}=0$, and $\bar{E}_{l(k j)}^{k}=\frac{3}{2} \bar{q}_{l j}$. Let us define generators

$T_{a}(x)=\frac{1}{\hbar \delta(0)}\left(\sigma_{a}\right)_{i}^{j} \bar{\pi}_{j}^{i}(x)$, where $\left(\sigma_{a}\right)_{j}^{i}$ for $a=1,2,3$ denote the Pauli spin matrices. Then contracting the middle relation of (1) with $\left(\sigma_{a}\right)_{k}^{l}$ we get the following relation

$$
\left[\bar{q}_{i j}(x), T_{a}(y)\right]=i\left(\sigma_{a}\right)_{(i}^{l} \bar{q}_{l j)} \frac{\delta(x-y)}{\delta(0)},
$$


namely that the two-metric $\bar{q}_{i j}$ transforms in the spin-two irreducible representation of $S U(2)$. Contraction of the last relation of (1) with $\left(\sigma_{a}\right)_{i}^{j}\left(\sigma_{b}\right)_{k}^{l}$ yields the relation

$$
\left[T_{a}(x), T_{b}(y)\right]=\frac{i}{2} \epsilon_{a b c} T_{c} \frac{\delta(x-y)}{\delta(0)},
$$

which is the $S U(2)$ Lie algebra with structure constants $\epsilon_{a b c}$. Hence the momentric variables in the $2+1$ formulation of ITQG transform, as a triplet of $S U$ (2) generators, in the adjoint representation of $S U(2)$.

Having conferred upon the fundamental variables a $S U(2)$ Lie-algebraic interpretation, it is next of interest to determine the $S U(2)$ invariants and their physical interpretation. From the Fierz identity for $S U(2)$

$$
\left(\sigma_{a}\right)_{j}^{i}\left(\sigma_{a}\right)_{l}^{k}=\frac{1}{2}\left(\delta_{l}^{i} \delta_{j}^{k}-\frac{1}{2} \delta_{j}^{i} \delta_{l}^{k}\right)
$$

We have, upon carrying out a contraction with $\bar{\pi}_{i}^{j} \bar{\pi}_{k}^{l}$, the following relation

$$
(\hbar \delta(0))^{2} T_{a} T_{a}=\frac{1}{2} \bar{\pi}_{i}^{j} \bar{\pi}_{j}^{i} \equiv \Delta,
$$

namely that the quadratic Casimir invariant for $S U(2)$ is proportional to the traceless modes of the Wheeler-DeWitt kinetic operator, forming the Hamiltonian for the free theory.

The dynamics of the evolution of the basic variables of the theory with respect to $T$ and the gauge invariant part of the intrinsic time $\ln q^{1 / 3}$ are encoded in the Physical Hamiltonian which we define as $H_{P h y s}=\frac{1}{\beta} \int_{\Sigma} \mathrm{d}^{3} x \sqrt{\Delta}$, and a unitary evolution operator

$$
U\left(T, T_{0}\right)=T \exp \left[\frac{i}{\hbar} \int_{T_{0}}^{T} H_{\text {Phys }}\left(T^{\prime}\right) \delta T^{\prime}\right] .
$$

This acts on a basis of states forming a separate $S U(2)$ multiplet at each spatial point $\prod_{x}|m, l\rangle_{x}$, satisfying the $S U(2)$ angular momentum relations

$$
T_{3}(x) \prod_{y}|m, l\rangle_{y}=m \prod_{y}|m, l\rangle_{y} ; \sqrt{\Delta} \prod_{y}|m, l\rangle_{y}=\hbar \delta(0) \sqrt{l(l+1)} \prod_{y}|m, l\rangle_{y} .
$$

One can construct from this a basis of $S U(2)$ spin-network states, with the $S U$ (2) singlets being diffeomorphism invariant. In this analogy to Loop Quantum Gravity, these states must be deemed to be in a momentum space representation.

\section{The BTZ Black Hole in Intrinsic Quantum Gravity}

Let us consider the metric for a BTZ black hole

$$
\mathrm{d} s^{2}=g_{00} \mathrm{~d} t^{2}+g_{r r} \mathrm{~d} r^{2}+g_{\varphi \varphi}\left(\mathrm{d} \varphi+N^{\varphi} \mathrm{d} t\right)^{2},
$$

where we have defined

$$
g_{00}=-N^{2}=-\left(-M+\frac{r^{2}}{l^{2}}+\frac{j^{2}}{4 r^{2}}\right) ; g_{r r}=\frac{1}{N^{2}} ; g_{\varphi \varphi}=r^{2} ; N^{\varphi}=-\frac{j}{2 r^{2}} ; N^{r}=0 \text {. }
$$


We will now put the metric (8) into the language of ITQG by calculating the fundamental basic variables of the theory. From the determinant of the spatial two-metric $q=q_{r r} q_{\varphi \varphi}=\frac{r^{2}}{-M+\frac{r^{2}}{l^{2}}+\frac{j^{2}}{4 r^{2}}}$, we can construct the unimodular spatial two-metric

$$
\bar{q}_{i j}=q_{i j} q^{-1 / 2}=\left(\begin{array}{cc}
\frac{1}{r}\left(-M+\frac{r^{2}}{l^{2}}+\frac{j^{2}}{4 r^{2}}\right)^{-1 / 2} & 0 \\
0 & r\left(-M+\frac{r^{2}}{l^{2}}+\frac{j^{2}}{4 r^{2}}\right)^{1 / 2}
\end{array}\right)
$$

with $\operatorname{det} \bar{q}_{i j}=1$. Next, we will construct the momentric variables $\bar{\pi}_{j}^{i}$. The extrinsic curvature is given by $K_{i j}=\frac{1}{2 N}\left(-\dot{q}_{i j}+\nabla_{i} N_{j}+\nabla_{j} N_{i}\right)$. We will need the lowered index form of the shift vector

$$
N_{r}=q_{r r} N^{r}+q_{r \varphi} N^{\varphi}=0 ; \quad N_{\varphi}=q_{\varphi r} N^{r}+q_{\varphi \varphi} N^{\varphi}=r^{2}\left(-\frac{1}{2 r^{2}}\right)=-\frac{j}{2} .
$$

Since the three-metric is time independent, then $\dot{g}_{\mu \nu}=0$ and only the covariant derivatives of the shift vector components will contribute

$$
K_{r r}=\frac{1}{N} \nabla_{r} N_{r}=0, \quad K_{\varphi \varphi}=\frac{1}{N} \nabla_{\varphi} N_{\varphi}=0 .
$$

For the covariant derivatives we have

$$
\begin{gathered}
\nabla_{r} N_{\varphi}=\partial_{r} N_{\varphi}-\Gamma_{r \varphi}^{r} N_{r}-\Gamma_{\varphi r}^{\varphi} N_{\varphi}=\partial_{r}(j / 2)-0-\frac{\partial_{r} q_{\varphi \varphi}}{2 q_{\varphi \varphi}} N_{\varphi}=\frac{\partial_{r} r^{2}}{2 r^{2}} \frac{j}{2}=\frac{j}{2 r} \\
\nabla_{\varphi} N_{r}=\partial_{\varphi} N_{r}-\Gamma_{\varphi r}^{r} N_{r}-\Gamma_{\varphi r}^{\varphi} N_{\varphi}=0-0+\frac{j}{2 r}
\end{gathered}
$$

Using $N=\sqrt{-M+\frac{r^{2}}{l^{2}}+\frac{j^{2}}{4 r^{2}}}$ and substituting (13) and (12), we have $K_{r \varphi}=\frac{j}{2 N r}$ with the following representation

$$
K_{i j}=\frac{j}{2 r}\left(-M+\frac{r^{2}}{l^{2}}+\frac{j^{2}}{4 r^{2}}\right)^{-1 / 2}\left(\begin{array}{ll}
0 & 1 \\
1 & 0
\end{array}\right) .
$$

The contravariant form is given by

$$
K^{i j}=q^{i m} K_{m n} q^{m j}=\frac{j}{2 r}\left(-M+\frac{r^{2}}{l^{2}}+\frac{j^{2}}{4 r^{2}}\right)^{-1 / 2}\left(\begin{array}{ll}
0 & 1 \\
1 & 0
\end{array}\right),
$$

which after the matrix multiplication yields the same components as $K_{i j}$. To get the momentric variables we must first compute the traceless form of $K^{i j}$, which is already traceless. In the ADM metric theory, we denote spatial 3-metric and its barred conjugate momentum on a spatial slice $\Sigma$ of four dimensional spacetime of topology $M=\Sigma \times R$ by $q_{i, j}$ and $\tilde{\pi}^{i j}$ respectively. The ADM conjugate 
momentum is given by $\tilde{\pi}^{i j}=\frac{1}{2} \sqrt{q}\left(K^{i j}-q^{i j} K\right)=\frac{1}{2} \sqrt{q} K^{i j}$. These leads to the barred momentum

$$
\bar{\pi}^{i j}=q^{1 / 2}\left(\tilde{\pi}^{i j}-\frac{1}{2} q^{i j} \tilde{\pi}\right)=\frac{q}{2} K^{i j},
$$

with matrix representation

$$
\begin{aligned}
& \tilde{\pi}_{i}^{j}=\bar{q}_{i m} \bar{\pi}^{m j} \\
& =\frac{j r}{4}\left(-M+\frac{r^{2}}{l^{2}}+\frac{j^{2}}{4 r^{2}}\right)^{-3 / 2}\left(\begin{array}{cc}
0 & \frac{1}{r}\left(-M+\frac{r^{2}}{l^{2}}+\frac{j^{2}}{4 r^{2}}\right)^{-1 / 2} \\
r\left(-M+\frac{r^{2}}{l^{2}}+\frac{j^{2}}{4 r^{2}}\right)^{1 / 2} & 0
\end{array}\right)
\end{aligned}
$$

\section{P-V Criticality in Modified BTZ Black Hole in Intrinsic Quantum Gravity}

To modify the BTZ black hole metric, we have from (10), $\bar{q}_{i j}=\frac{1}{g(r)}$ where

$$
g(r)=r\left(-M+\frac{r^{2}}{l^{2}}+\frac{j^{2}}{4 r^{2}}\right)^{1 / 2}+h(r, p)
$$

and $h(r, p)$ is function that must be determined. After differentiation and further simplification we get

$$
\begin{aligned}
g^{\prime}(r)= & -M^{1 / 2}+\left(\frac{M^{-1 / 2} r^{2}}{2}-M^{1 / 2} r^{2}\right) \frac{1}{l^{2}}+\left(\frac{M^{-1 / 2}}{2}+M^{1 / 2}\right) \frac{j^{2}}{4 r^{2}} \\
& -\frac{M^{-1 / 2}}{2}\left(\frac{r^{2}}{l^{2}}\right)^{2}+\frac{M^{-1 / 2}}{2}\left(\frac{j^{2}}{4 r^{2}}\right)^{2}+h^{\prime}(r, p)
\end{aligned}
$$

But the position of the black hole event horizon is determined by the larger root of $g\left(r_{+}\right)=0$, and by using the Euclidean trick we have the black hole temperature given by

$$
\begin{aligned}
T & =\frac{1}{\beta}=\frac{g^{\prime}\left(r_{+}\right)}{4 \pi} \\
= & -\frac{M^{1 / 2}}{4 \pi}+\frac{1}{4 \pi}\left(\frac{M^{-1 / 2} r^{2}}{2}-M^{1 / 2} r^{2}\right) \frac{8 \pi}{3} P+\frac{1}{4 \pi}\left(\frac{M^{-1 / 2}}{2}+M^{1 / 2}\right) \frac{j^{2}}{4 r^{2}} \\
& -\frac{1}{4 \pi} \frac{M^{-1 / 2} r^{4}}{2}\left(\frac{8 \pi}{2}\right)^{2} P^{2}+\frac{M^{-1 / 2}}{2} \frac{1}{4 \pi}\left(\frac{j^{2}}{4 r^{2}}\right)^{2}+h^{\prime}(r, p)
\end{aligned}
$$

where in the case of the asymptotically BTZ black hole the pressure $P$ is

$$
\begin{aligned}
P & =\frac{-1}{8 \pi} \Lambda=\frac{3}{8 \pi} \frac{1}{l^{2}}, \\
& \Rightarrow \frac{8 \pi}{3} P=\frac{1}{l^{2}} .
\end{aligned}
$$

From the Wan der Waals relation 


$$
T=\left(P-\frac{a}{v^{2}}\right)(v+b)=P v-P b+\frac{a}{v}-\frac{a b}{v^{2}},
$$

with

$$
v=2 r,
$$

we have

$$
T=2 P r_{+}-P b+\frac{a}{2 r_{+}}-\frac{a b}{4 r_{+}^{2}} .
$$

Now in order to find $h(r, P)$ we use the anstaz

$$
h(r, P)=A(r)+P B(r, P) \text {, }
$$

with

$$
h^{\prime}(r, P)=A^{\prime}(r)+P B^{\prime}(r, P) .
$$

So from (20), (24) and (26) we have

$$
\begin{aligned}
& \frac{-M^{1 / 2}}{4 \pi}+\left(\frac{M^{-1 / 2} r_{+}^{2}}{2}-M^{1 / 2} r_{+}^{2}\right) \frac{2}{3} P+\left(\frac{M^{-1 / 2}}{2}+M^{1 / 2}\right) \frac{j^{2}}{4 r_{+}^{2}} \frac{1}{4 \pi} \\
& -\frac{M^{-1 / 2}}{2} \frac{r_{+}^{4}}{4 \pi}\left(\frac{8 \pi}{3}\right)^{2} P^{2}+\frac{M^{-1 / 2}}{2}\left(\frac{j^{2}}{4 r_{+}^{2}}\right)^{2} \frac{1}{4 \pi}+\frac{A^{\prime}(r)}{4 \pi}+\frac{P^{\prime} B(r)}{4 \pi} \\
& -2 P r_{+}+P b-\frac{a}{2 r_{+}}+\frac{a b}{4 r_{+}^{2}}=0 .
\end{aligned}
$$

From which we have the following two independent equations

$$
\begin{gathered}
\left(\frac{M^{-1 / 2} r_{+}^{2}}{2}-M^{1 / 2} r_{+}^{2}\right) \frac{2 P}{3}-\frac{M^{-1 / 2}}{2} r_{+}^{4}\left(\frac{8 \pi}{3}\right)^{2} \frac{P^{2}}{4 \pi}+\frac{P B^{\prime}(r)}{4 \pi}-2 P r_{+}+P b=0, \\
-\frac{M^{1 / 2}}{4 \pi}+\frac{1}{4 \pi}\left(\frac{M^{-1 / 2}}{2}+M^{1 / 2}\right) \frac{j^{2}}{4 r_{+}^{2}}+\frac{M^{-1 / 2}}{2} \frac{1}{4 \pi}\left(\frac{j^{2}}{4 r_{+}^{2}}\right)^{2}+\frac{A^{\prime}(r)}{4 \pi}-\frac{a}{2 r_{+}}+\frac{a b}{4 r_{+}^{2}}=0,
\end{gathered}
$$

for which we are able to solve for $A(r)$ and $B(r)$ as follows

$$
\begin{gathered}
A(r)=4 \pi \frac{a}{2} \ln \left(r_{+}\right)+\pi a b \frac{1}{r_{+}}+M^{1 / 2} r_{+}+\left(\frac{M^{-1 / 2}}{2}+M^{1 / 2}\right) \frac{j^{2}}{4 r_{+}}-\frac{M^{-1 / 2}}{6}\left(\frac{j^{2}}{4}\right)^{2} \frac{1}{r_{+}^{3}}, \\
B(r)=4 \pi r_{+}^{2}-4 \pi b r_{+}+M^{-1 / 2} \frac{r_{+}^{5}}{10}\left(\frac{8 \pi}{3}\right)^{2} P-\left(\frac{M^{-1 / 2} r_{+}^{3}}{6}-\frac{M^{1 / 2} r_{+}^{3}}{3}\right) \frac{8 \pi}{3} .
\end{gathered}
$$

But putting the above results for $A(r)$ and $B(r)$ into (25) and then put the resulting explicit expression for $h(r, P)$ into (18), and using (22) we get the modified expression for $g(r)$ given by

$$
\begin{aligned}
g(r)= & r\left(-M+\frac{r^{2}}{l^{2}}+\frac{j^{2}}{4 r^{2}}\right)^{1 / 2}+2 \pi a \ln (r)+\frac{\pi a b}{r}+M^{1 / 2} r \\
& +\left(\frac{M^{-1 / 2}}{2}+M^{1 / 2}\right) \frac{j^{2}}{4 r}-\frac{M^{-1 / 2}}{6 r^{3}}\left(\frac{j^{2}}{4}\right)^{2}+\frac{3 r^{2}}{2 l^{2}}-\frac{3 b r}{2 l^{2}} \\
& +\frac{M^{-1 / 2} r^{5}}{10 l^{4}}-\left(\frac{M^{-1 / 2} r^{3}}{6}-\frac{M^{1 / 2}}{3} r^{3}\right) \frac{1}{l^{2}}
\end{aligned}
$$


which gives us the modified BTZ black hole from which

$$
\begin{aligned}
& T=\left.\frac{1}{4 \pi} g^{\prime}(r)\right|_{r=r+} \\
& =\frac{M^{-1 / 2} r^{2} P}{3}-\frac{2 M^{1 / 2} r^{2} P}{3}+\frac{a}{2 r}-\frac{a b}{4 r^{2}}+2 P r-b P-\left(\frac{M^{-1 / 2} r^{2}}{2}-M^{1 / 2} r^{2}\right) \frac{2 P}{3}
\end{aligned}
$$

thus solving for the pressure $P$ and further simplifications we get

$$
P=\frac{1}{[-b+2 r]}\left[T-\frac{a}{2 r}+\frac{a b}{4 r^{2}}\right] \text {. }
$$

From the above, we observe that Equation (32) was also derived in [1] in another different formalism of quantum gravity. Thus we have shown that Equation (32) must be universal for the modified BTZ black hole irrespective of the formalism used. Thus continuing further we are then able to find the BTZ black hole critical points $T_{c}, P_{c}$, and $V_{c}$ as

$$
\begin{gathered}
V_{c}=3 b, \\
P_{c}=\frac{a}{27 b^{2}}
\end{gathered}
$$

and

$$
T_{c}=\frac{8 a}{27 b}
$$

using the conditions

$$
\frac{\partial P}{\partial V}=0 \text { and } \frac{\partial^{2} P}{\partial V^{2}}=0 .
$$

Then finally from (33) we are able to find an interesting relation which is exactly the same as for the Van der Waals fluid called the thermodynamic critical compressibility factor and it is universal number predicted for the modified BTZ black hole [1].

$$
\frac{P_{c} V_{c}}{T_{c}}=\frac{3}{8}
$$

Therefore from above results, we conclude that the modified BTZ black hole also exhibit the thermodynamic P-V criticality behavior in ITQG framework.

\section{Conclusion}

It is a well known fact that usual BTZ black does not have the critical behavior in $P_{c}, T_{c}, V_{c}$. But in order for the black hole to have these behaviors, it needs to be modified and we do the transformation by using a suitable anstaz as function $h(r, p)$. In our case, we modified the BTZ black hole within the frame work of ITQG formalism and we showed that indeed the modified BTZ black hole exhibits the P-V criticality and thus the results are the same as that of the Van der Waals fluid. 


\section{Acknowledgements}

ASK would like to thank Prof. Eyo Eyo Ita III for the advices and discussions regarding the problem and my employer the University of South Africa for providing me with the conducive environment and facilities to conduct the research of this paper.

\section{Data Availability}

No data was used to support this study.

\section{Conflicts of Interest}

The author declares that there are no conflicts of interest regarding the publication of this paper.

\section{References}

[1] Sadeghi, J. and Kubeka, A.S. (2016) International Journal of Theoretical Physics, 55, 2455-2459. https://doi.org/10.1007/s10773-015-2882-x

[2] Sadeghi, J., Pourhassan, B. and Rostami, M. (2016) Physical Review D, 94, 064006. https://doi.org/10.1103/PhysRevD.94.064006

[3] Ita, E.E., Soo, C. and Yu, H.-L. (2015) Prog. Theor. Exp. Phys., 083 E01.

[4] DeWitt, B.S. (1967) Physical Review Journals Archive, 160, 1113. https://doi.org/10.1103/PhysRev.160.1113

[5] Dirac, P.A.M. (1958) Proceedings of the Royal Society of London A, 246, 333

[6] Klauder, J.R. (2006) International Journal of Geometric Methods in Modern Physics, 3, 81-94. https://doi.org/10.1142/S0219887806001028

[7] Lin, C.Y. and Soo, C. (2009) Physics Letters B, 671, 493-495. https://doi.org/10.1016/j.physletb.2008.12.051

[8] Murchadha, N.O., Soo, C. and Yu, H.-L. (2013) Class. Quantum Grav, 30, 095016. https://doi.org/10.1088/0264-9381/30/9/095016

[9] Soo, C. and Yu, H.-L. (2014) Prog. Theor. Phys, 2014, $013 \mathrm{E} 01$.

[10] Wheeler, J.A. (1968) Superpsace and the Nature of Quantum Geometrodynamics, in Battelle Rencontres, 1967. In: DeWitt, C.M. and Wheeler, J.A., Eds., Lectures in Mathematics and Physics, W. A. Benjamin, New York.

[11] Ita, E.E. and Soo, C. (2015) Annals of Physics, 359, 80-96. https://doi.org/10.1016/j.aop.2015.04.016

[12] Ita, E.E. and Kubeka, A.S. (2016) Universe, 2, 18. https://doi.org/10.3390/universe2030018 